\title{
Constructions of algebraic lattices
}

\author{
A.A. ANDRADE ${ }^{1}$, A.J. FERRARI ${ }^{2}$, C.W.O. BENEDITO ${ }^{3}$ and S.I.R. COSTA ${ }^{4}$
}

1,3 Department of Mathematics, IBILCE, UNESP, 15054-000 São José do Rio Preto, SP, Brazil

${ }^{2}$ Department of Applied Mathematics, IMECC, UNICAMP, 13083-859 Campinas, SP, Brazil

${ }^{4}$ Department of Mathematics, IMECC, UNICAMP, 13083-859 Campinas, SP, Brazil

E-mails: andrade@ibilce.unesp.br / ferrari@ime.unicamp.br /

cwinktc@hotmail.com / sueli@ime.unicamp.br

\begin{abstract}
In this work we present constructions of algebraic lattices in Euclidean space with optimal center density in dimensions $2,3,4,6,8$ and 12, which are rotated versions of the lattices $\Lambda_{n}$, for $n=2,3,4,6,8$ and $K_{12}$. These algebraic lattices are constructed through twisted canonical homomorphism via ideals of a ring of algebraic integers.
\end{abstract}

Mathematical subject classification: $18 \mathrm{~B} 35,94 \mathrm{~A} 15,20 \mathrm{H} 10$.

Key words: algebraic lattice, algebraic number field, center density, twisted canonical homomorphism.

\section{Introduction}

The classical sphere packing problem, still unsolved even today, is to find out how densely a large number of identical spheres can be packed together. To state this another way, consider a large empty region, such as an aircraft hangar, and ask what is the greatest number of ball bearings that can be packed into this region. If instead of ball bearings we try to pack identical wooden cubes the answer becomes easy. But spheres do not fit together so well as cubes, and there is always some wasted space in between. No matter how cleverly the ball bearings are arranged, about one quarter of the space will not be used. 
Elementary number theory has been very useful to the development of error correcting codes in the early age of coding theory and the theory of Euclidian lattices became of great interest for the design of dense signal constellations well suited for transmission over AWGN channel. Furthermore, algebraic number theory has been very useful in mathematical tool that enables the design of good coding schemes for the fading channels (wireless communications). Algebraic lattices defined over algebraic number fields have been studied in several papers and from different points of view [1-7]. Giraud and Belfiore [8] proposed a technique for constructing signal sets suitable for the Rayleigh fading channel. The basic idea was to use lattice rotations to increase diversity, that is, the number of different values in the components of any two distinct points of the constellation. Boutros et al. [9] constructed rotated versions of lattices $D_{4}, K_{12}$ and $\Lambda_{6}$ via ideals of $\mathbb{Q}\left(\zeta_{n}\right)$, for $n=8,21$ and 40, respectively. Bayer-Fluckiger [1-7] constructed rotated versions of lattices $A_{p-1}$, where $p$ is an odd prime number, $D_{4}, E_{6}, E_{8}, K_{12}, \Lambda_{24}$ and Craig's lattices $A_{p}^{(k)}$. Thus, having the construction of rotated lattices as our goal, in this work we present algebraic lattices in Euclidean space with optimal center density in dimensions 2, 3, 4, 6, 8 and 12 making use of the twisted canonical homomorphism.

This work is organized as follows. In Section 2, we present basic results of algebraic number fields and lattices. In Section 3, we present results about algebraic lattices making use of the twisted canonical homomorphism $\sigma_{\alpha}$ and examples of algebraic lattices with optimal center density in dimensions 2, 3, 4, 6, 8 and 12. Finally, in Section 4, we give ours conclusions.

\section{Basic results}

In this section, we present some results of algebraic number theory and lattices $[10,11]$ that are important to the development of the next section.

\subsection{Algebraic number theory}

Let $\mathbb{L}$ be an algebraic number field, i.e., $\mathbb{L}$ is an algebraic extension of $\mathbb{Q}$ of finite degree $n$, and therefore $[\mathbb{L}: \mathbb{Q}]=n$. 
Definition 2.1. An element $\beta \in \mathbb{L}$ is called an algebraic integer if there exists a monic polynomial non-zero $f(x)$ with coefficients in $\mathbb{Z}$ such that $f(\beta)=0$. The set $\mathcal{O}_{\mathbb{L}}=\{\beta \in \mathbb{L}: \beta$ is an algebraic integer $\}$ is a ring called ring of algebraic integers in $\mathbb{L}$.

Let $\mathcal{O}_{\mathbb{L}}$ be the ring of algebraic integers of $\mathbb{L}$. It can be shown that $\mathcal{O}_{\mathbb{L}}$ has a basis $\left\{x_{1}, x_{2}, \ldots, x_{n}\right\}$ over $\mathbb{Z}$ called an integral basis of $\mathbb{L}$.

Definition 2.2. $\quad$ Let $\mathcal{A}$ be a non-zero ideal of $\mathcal{O}_{\mathbb{L}}$. The norm of the ideal $\mathcal{A}$ is defined as the number of elements of the quotient ring $\mathcal{O}_{\mathbb{L}} / \mathcal{A}$, i.e., $\mathcal{N}_{\mathbb{L}}(\mathcal{A})=$ $\left|\mathcal{O}_{\mathbb{L}} / \mathcal{A}\right|$.

Definition 2.3. Let $\sigma_{1}, \sigma_{2}, \ldots, \sigma_{n}$ be the monomorphisms of $\mathbb{L}$ in $\mathbb{C}$. The trace and the norm of an element $\beta \in \mathbb{L}$ over $\mathbb{Q}$ are defined, respectively, by

$$
\operatorname{Tr}_{\mathbb{L}}(\beta)=\sum_{i=1}^{n} \sigma_{i}(\beta) \text { and } \mathcal{N}_{\mathbb{L}}(\beta)=\prod_{i=1}^{n} \sigma_{i}(\beta) .
$$

The discriminant of $\mathbb{L}$ over $\mathbb{Q}$ is defined by

$$
\mathcal{D}_{\mathbb{L}}=\mathcal{D}\left(x_{1}, x_{2}, \ldots, x_{n}\right)=\operatorname{det}_{1 \leq i, j \leq n}\left(\sigma_{i}\left(x_{j}\right)\right)^{2},
$$

where $\left\{x_{1}, x_{2}, \ldots, x_{n}\right\}$ is an integral basis of $\mathbb{L}$.

\subsection{Packing lattice}

In this section we present basic facts about packing lattices. The classical problem of the sphere packing consists in to find identical spheres in $\mathbb{R}^{n}$ such that the proportion of the space that is occupied by the spheres is optimal.

Definition 2.4. An additive subgroup $\Lambda \subseteq \mathbb{R}^{n}$ is a lattice if there exists a basis $B=\left\{x_{1}, x_{2}, \cdots, x_{n}\right\}$ in $\mathbb{R}^{n}$ such that

$$
\Lambda=\left\{x \in \mathbb{R}^{n}: x=\sum_{i=1}^{n} a_{i} x_{i}, \text { with } a_{i} \in \mathbb{Z}, \text { for } i=1,2, \cdots, n\right\} .
$$

The set $B$ is called a $\mathbb{Z}$-basis of the lattice $\Lambda$.

Let $\Lambda \subset \mathbb{R}^{n}$ be a lattice with $\mathbb{Z}$-basis $B=\left\{x_{1}, x_{2}, \cdots, x_{n}\right\}$. 
Definition 2.5. The set

$$
\mathcal{P}_{B}=\left\{x \in \mathbb{R}^{n}: x=\sum_{i=1}^{n} \lambda_{i} x_{i}, 0 \leq \lambda_{i}<1, \text { for } i=1,2, \cdots, n\right\},
$$

is called fundamental region of $\Lambda$ with respect to basis $B$.

Definition 2.6. The volume of the lattice $\Lambda$ is defined as the module of determinant of the matrix $M=\left(x_{i j}\right)_{i, j=1}^{n}$, where $x_{i}=\left(x_{i 1}, x_{i 2}, \cdots, x_{i n}\right)$, for $i=1,2, \cdots, n$, and denoted by $\operatorname{Vol}(\Lambda)=|\operatorname{det}(M)|$. The matrix $M$ is called a generator matrix for the lattice $\Lambda$.

For obtain optimal packing lattices we need to find spheres whose centers are points of a lattice $\Lambda$ and that the intersection of any two spheres is only a point. For the determination of the radius of these spheres, note that fixed $k>0$, the intersection of the compact set $\left\{x \in \mathbb{R}^{n} ;|x| \leq k\right\}$ with the lattice $\Lambda$ is a finite set. Therefore the number $t=\min \{|x| ; x \in \Lambda, x \neq 0\}$ is well defined. Furthermore, $\rho(\Lambda)=t / 2$ is the greatest radius such that it is possible to obtain a packing lattice. If $\mathcal{B}(\rho(\Lambda))$ is the sphere with center in the origin and radius $\rho(\Lambda)$ then the packing density of $\Lambda$ is defined by

$$
\Delta(\Lambda)=\frac{\operatorname{Vol}(\mathcal{B}(\rho(\Lambda)))}{\operatorname{Vol}(\Lambda)}=\operatorname{Vol}(\mathcal{B}(1)) \rho(\Lambda)^{n} \frac{1}{\operatorname{Vol}(\Lambda)},
$$

where $\delta(\Lambda)=\rho(\Lambda)^{n} / \mathcal{V o l}(\Lambda)$ is called the center density of the lattice $\Lambda$.

\section{Algebraic lattice}

The connections between lattices and algebraic number fields have been studied by many authors from Minkowski onwards [1-7]. In this section, we define the twisted canonical homomorphism $[3,5]$, and we present some results about algebraic lattices. Furthermore, we present some examples of algebraic lattices in $\mathbb{R}^{n}$ with optimal center density.

Let $\mathbb{L}$ be an algebraic number field of degree $n$ and $\mathcal{O}_{\mathbb{L}}$ be the ring of algebraic integers of $\mathbb{L}$. Let $\sigma_{j}: \mathbb{L} \rightarrow \mathbb{C}$ be the $n$ distinct monomorphisms of $\mathbb{L}$. If $\sigma_{j}(\mathbb{L}) \subseteq \mathbb{R}$, say that $\sigma_{j}$ is real, case contrary, $\sigma_{j}$ is called imaginary. If all the monomorphisms are reals, $\mathbb{L}$ is called a totally real field and if all the monomorphisms are imaginary, $\mathbb{L}$ is called a totally complex field. If $\varphi: \mathbb{C} \rightarrow \mathbb{C}$ is the 
complex conjugation then for all $j=1,2, \ldots, n$, it follows that $\varphi \circ \sigma_{j}=\sigma_{k}$, for some $k=1,2, \ldots, n$, and that $\sigma_{j}=\sigma_{k}$ if and only if $\sigma_{j}(\mathbb{L}) \subset \mathbb{R}$. Hence if $r_{1}$ is the number of indices such that $\sigma_{j}(\mathbb{L}) \subset \mathbb{R}$, we can ordered the monomorphisms $\sigma_{1}, \sigma_{2}, \ldots, \sigma_{n}$ of such manner that $\sigma_{1}, \sigma_{2}, \ldots, \sigma_{r_{1}}$ are the real monomorphisms and that $\sigma_{r_{1}+r_{2}+j}=\overline{\sigma_{r_{1}+j}}$, for $j=1, \ldots, r_{2}$. Hence $n-r_{1}$ is an even number and it can be write as $r_{1}+2 r_{2}=n$.

Definition 3.1 $[3,5]$. The twisted canonical homomorphism $\sigma_{\alpha}: \mathbb{L} \longrightarrow \mathbb{R}^{n}$ is defined by

$$
\begin{aligned}
\sigma_{\alpha}(x)= & \left(\sqrt{\alpha_{1}} \sigma_{1}(x), \ldots, \sqrt{\alpha_{r_{1}}} \sigma_{r_{1}}(x), \Re\left(\sqrt{\alpha_{r_{1}+1}} \sigma_{r_{1}+1}(x)\right), \ldots\right. \\
& \left.\ldots, \Re\left(\sqrt{\alpha_{r_{1}+r_{2}}} \sigma_{r_{1}+r_{2}}(x)\right), \Im\left(\sqrt{\alpha_{r_{1}+r_{2}}} \sigma_{r_{1}+r_{2}}(x)\right)\right),
\end{aligned}
$$

where $\alpha, x \in \mathbb{L}, \sigma_{i}(\alpha) \in \mathbb{R}$ and $\alpha_{i}=\sigma_{i}(\alpha)>0$, for $i=1,2, \ldots, r_{1}+r_{2}$, and the notations $\Re(\beta)$ and $\Im(\beta)$ are the real and imaginary parts of the complex number $\beta$, respectively.

In the Definition 3.1 taking $\alpha=1$ we have that $\sigma_{1}$ is the canonical homomorphism (or Minkowski) [10]. By Bayer-Fluckiger [3], it follows that $\sigma_{\alpha}(\mathcal{A})$ is an algebraic lattice in $\mathbb{R}^{n}$, where $\mathcal{A} \subseteq \mathcal{O}_{\mathbb{L}}$ is an ideal. By Samuel [10] it follows that the volume of $\sigma_{\alpha}(\mathcal{A})$ is given by

$$
\mathcal{V o l}\left(\sigma_{\alpha}(\mathcal{A})\right)=\sqrt{\alpha_{1} \alpha_{2} \ldots \alpha_{r_{1}}} \alpha_{r_{1}+1} \alpha_{r_{1}+2} \ldots \alpha_{r_{1}+r_{2}}(2 i)^{-r_{2}}\left|\operatorname{det}_{1 \leq j, k \leq n}\left(\sigma_{j}\left(x_{k}\right)\right)\right|,
$$

where $x_{1}, x_{2}, \cdots, x_{n}$ is a $\mathbb{Z}$-basis of $\mathcal{A}$. Furthermore, if $\mathbb{L}$ is a totally real field (or totally complex) then the volume of $\sigma_{\alpha}(\mathcal{A})$ is given by

$$
\mathcal{V o l}\left(\sigma_{\alpha}(\mathcal{A})\right)=2^{-r_{2}} \mathcal{N}_{\mathbb{L}}(\alpha)^{\frac{1}{2}}\left|\operatorname{det}_{1 \leq j, k \leq n}\left(\sigma_{j}\left(x_{k}\right)\right)\right| .
$$

Let $\mathbb{L}$ be a totally real number field (or totally complex) of finite degree $n, \mathcal{D}_{\mathbb{L}}$ be the discriminant of $\mathbb{L}$ and $\mathcal{O}_{\mathbb{L}}$ be the ring of algebraic integers in $\mathbb{L}$.

Proposition 3.2 [10]. If $\mathcal{A}$ is a non-zero ideal of $\mathcal{O}_{\mathbb{L}}$ then the volume of $\sigma_{\alpha}\left(\mathcal{O}_{\mathbb{L}}\right)$ and the volume of $\sigma_{\alpha}(\mathcal{A})$ are given, respectively, by

$$
\begin{aligned}
& \operatorname{Vol}\left(\sigma_{\alpha}\left(\mathcal{O}_{\mathbb{L}}\right)\right)=2^{-r_{2}}\left|\mathcal{N}_{\mathbb{L}}(\alpha) \mathcal{D}_{\mathbb{L}}\right|^{\frac{1}{2}} \quad \text { and } \\
& \operatorname{Vol}\left(\sigma_{\alpha}(\mathcal{A})\right)=2^{-r_{2}}\left|\mathcal{N}_{\mathbb{L}}(\alpha) \mathcal{D}_{\mathbb{L}}\right|^{\frac{1}{2}} \mathcal{N}_{\mathbb{L}}(\mathcal{A}) .
\end{aligned}
$$


Proof. We have that $\operatorname{Vol}\left(\sigma_{\alpha}\left(\mathcal{O}_{\mathbb{L}}\right)\right)=2^{-r_{2}}\left|\mathcal{N}_{\mathbb{L}}(\alpha) \mathcal{D}_{\mathbb{L}}\right|^{\frac{1}{2}}$, because $\mathcal{D}_{\mathbb{L}}=$ $\operatorname{det}\left(\sigma_{i}\left(x_{k}\right)\right)^{2}$, where $\left\{x_{1}, x_{2}, \ldots, x_{n}\right\}$ is a $\mathbb{Z}$-basis of $\mathcal{O}_{\mathbb{L}}$. For the second formula, since $\mathcal{O}_{\mathbb{L}} / \mathcal{A}$ is isomorph to $\sigma_{\alpha}\left(\mathcal{O}_{\mathbb{L}}\right) / \sigma_{\alpha}(\mathcal{A})$ it follows that $\sigma_{\alpha}(\mathcal{A})$ is an additive subgroup of $\sigma_{\alpha}\left(\mathcal{O}_{\mathbb{L}}\right)$ of index $\mathcal{N}_{\mathbb{L}}(\mathcal{A})$. Furthermore, since a fundamental domain of $\sigma_{\alpha}(\mathcal{A})$ is a disjoint union of $\mathcal{N}_{\mathbb{L}}(\mathcal{A})$ copies of a fundamental domain of $\sigma_{\alpha}\left(\mathcal{O}_{\mathbb{L}}\right)$ it follows that $\operatorname{Vol}\left(\sigma_{\alpha}(\mathcal{A})\right)=\operatorname{Vol}\left(\sigma_{\alpha}\left(\mathcal{O}_{\mathbb{L}}\right)\right) \mathcal{N}_{\mathbb{L}}(\mathcal{A})$ and consequently $\mathcal{V o l}\left(\sigma_{\alpha}(\mathcal{A})\right)=2^{-r_{2}}\left|\mathcal{N}_{\mathbb{L}}(\alpha) \mathcal{D}_{\mathbb{L}}\right|^{\frac{1}{2}} \mathcal{N}_{\mathbb{L}}(\mathcal{A})$.

By Conway and Sloane [11] we have that if $x \in \mathbb{L}$ then

$$
\left|\sigma_{\alpha}(x)\right|^{2}=c_{\alpha} \operatorname{Tr}_{\mathbb{L}}(\alpha x \bar{x}),
$$

where $c_{\alpha}=1$ if $\mathbb{L}$ is a totally real field, $c_{\alpha}=\frac{1}{2}$ if $\mathbb{L}$ is a totally complex field and $\bar{x}$ is the complex conjugate of the element $x$. Therefore $\rho\left(\sigma_{\alpha}(\mathcal{A})\right)=$ $\frac{1}{2} \min \left\{\left|\sigma_{\alpha}(\mathcal{A})\right|: x \in \mathcal{A}, x \neq 0\right\}=\frac{1}{2} \min \left\{\sqrt{c_{\alpha} \operatorname{Tr}_{\mathbb{L}}(\alpha x \bar{x})}: x \in \mathcal{A}, x \neq 0\right\}$, where $\mathcal{A}$ is an ideal of $\mathcal{O}_{\mathbb{L}}$.

Proposition 3.3. If $\mathcal{A}$ is a non-zero ideal of $\mathcal{O}_{\mathbb{L}}$ then thecenter density of the lattice $\sigma_{\alpha}(\mathcal{A})$ is given by

$$
\delta\left(\sigma_{\alpha}(\mathcal{A})\right)=\frac{1}{2^{n}\left|\mathcal{N}_{\mathbb{L}}(\alpha) \mathcal{D}_{\mathbb{L}}\right|^{1 / 2}} \frac{t_{\alpha}{ }^{n / 2}}{\mathcal{N}_{\mathbb{L}}(\mathcal{A})},
$$

where $t_{\alpha}=\min \left\{\operatorname{Tr}_{\mathbb{L}}(\alpha x \bar{x}): x \in \mathcal{A}, x \neq 0\right\}$.

Proof. Let $t_{\alpha}=\min \left\{\operatorname{Tr}_{\mathbb{L}}(\alpha x \bar{x}), x \in \mathcal{A}, x \neq 0\right\}$. If $\mathbb{L}$ is a totally real field then

$$
\begin{aligned}
\delta\left(\sigma_{\alpha}(\mathcal{A})\right) & =\frac{\rho\left(\sigma_{\alpha}(\mathcal{A})\right)}{\operatorname{Vol}\left(\sigma_{\alpha}(\mathcal{A})\right)}=\frac{\left(\frac{\sqrt{t_{\alpha}}}{2}\right)^{n}}{\left|\mathcal{N}_{\mathbb{L}}(\alpha) \mathcal{D}_{\mathbb{L}}\right|^{\frac{1}{2}} \mathcal{N}_{\mathbb{L}}(\mathcal{A})} \\
& =\frac{\left(\sqrt{\frac{t_{\alpha}}{4}}\right)^{n}}{\left|\mathcal{N}_{\mathbb{L}}(\alpha) \mathcal{D}_{\mathbb{L}}\right|^{\frac{1}{2}} \mathcal{N}_{\mathbb{L}}(\mathcal{A})}=\frac{\left(\frac{t_{\alpha}}{4}\right)^{\frac{n}{2}}}{\left|\mathcal{N}_{\mathbb{L}}(\alpha) \mathcal{D}_{\mathbb{L}}\right|^{\frac{1}{2}} \mathcal{N}_{\mathbb{L}}(\mathcal{A})} \\
& =\frac{1}{2^{n}\left|\mathcal{N}_{\mathbb{L}}(\alpha) \mathcal{D}_{\mathbb{L}}\right|^{\frac{1}{2}}} \frac{t_{\alpha}{ }^{n / 2}}{\mathcal{N}_{\mathbb{L}}(\mathcal{A})}
\end{aligned}
$$


and if $\mathbb{L}$ is a totally complex field then

$$
\begin{aligned}
& \delta\left(\sigma_{\alpha}(\mathcal{A})\right)=\frac{\rho\left(\sigma_{\alpha}(\mathcal{A})\right)}{\operatorname{Vol}\left(\sigma_{\alpha}(\mathcal{A})\right)}=\frac{\left(\frac{\sqrt{\frac{1}{2} t_{\alpha}}}{2}\right)^{n}}{2^{\frac{-n}{2}}\left|\mathcal{N}_{\mathbb{L}}(\alpha) \mathcal{D}_{\mathbb{L}}\right|^{\frac{1}{2}} \mathcal{N}_{\mathbb{L}}(\mathcal{A})} \\
& =\frac{\frac{2^{\frac{n}{2}} t_{\alpha^{\frac{n}{2}}}}{2^{\frac{3 n}{2}}}}{\left|\mathcal{N}_{\mathbb{L}}(\alpha) \mathcal{D}_{\mathbb{L}}\right|^{\frac{1}{2}} \mathcal{N}_{\mathbb{L}}(\mathcal{A})}=\frac{\frac{t_{\alpha^{\frac{n}{2}}}}{2^{n}}}{\left|\mathcal{N}_{\mathbb{L}}(\alpha) \mathcal{D}_{\mathbb{L}}\right|^{\frac{1}{2}} \mathcal{N}_{\mathbb{L}}(\mathcal{A})} \\
& =\frac{\frac{t_{\alpha^{\frac{n}{2}}}}{(\sqrt{4})^{n}}}{\left|\mathcal{N}_{\mathbb{L}}(\alpha) \mathcal{D}_{\mathbb{L}}\right|^{\frac{1}{2}} \mathcal{N}_{\mathbb{L}}(\mathcal{A})}=\frac{\frac{t_{\alpha^{\frac{n}{2}}}}{4^{\frac{n}{2}}}}{\left|\mathcal{N}_{\mathbb{L}}(\alpha) \mathcal{D}_{\mathbb{L}}\right|^{\frac{1}{2}} \mathcal{N}_{\mathbb{L}}(\mathcal{A})} \\
& =\frac{\left(\frac{t_{\alpha}}{4}\right)^{\frac{n}{2}}}{\left|\mathcal{N}_{\mathbb{L}}(\alpha) \mathcal{D}_{\mathbb{L}}\right|^{\frac{1}{2}} \mathcal{N}_{\mathbb{L}}(\mathcal{A})}=\frac{1}{2^{n}\left|\mathcal{N}_{\mathbb{L}}(\alpha) \mathcal{D}_{\mathbb{L}}\right|^{\frac{1}{2}}} \frac{t_{\alpha}{ }^{n / 2}}{\mathcal{N}_{\mathbb{L}}(\mathcal{A})}
\end{aligned}
$$

Therefore the center density is the same in both cases.

Example 3.4. If $\mathbb{L}=\mathbb{Q}\left(\zeta_{6}\right)$, where $\zeta_{6}$ is a primitive 6-th root of unity, $\alpha=2$ and $\mathcal{A}=\left(1-\zeta_{6}\right) \mathcal{O}_{\mathbb{L}}$ is an ideal of $\mathcal{O}_{\mathbb{L}}=\mathbb{Z}\left[\zeta_{6}\right]$, then $[\mathbb{L}: \mathbb{Q}]=2, \mathcal{D}_{\mathbb{L}}=-3$, $\mathcal{N}_{\mathbb{L}}(\alpha)=4$ and $\mathcal{N}_{\mathbb{L}}(\mathcal{A})=1$. If $x \in \mathcal{A}$ then $x=\left(a_{0}+a_{1} \zeta_{6}\right)\left(1-\zeta_{6}\right)$, with $a_{0}, a_{1} \in \mathbb{Z}$, and thus $\operatorname{Tr}_{\mathbb{L}}(\alpha x \bar{x})=4\left(a_{0}^{2}+a_{1}^{2}+a_{0} a_{1}\right)$. Therefore, $t_{\alpha}=$ $\min \left\{\operatorname{Tr}_{\mathbb{L}}(\alpha x \bar{x}): x \in \mathcal{A}, x \neq 0\right\}=4$, with $a_{0}=1$ and $a_{1}=0$, and the center density of the lattice $\sigma_{\alpha}(\mathcal{A})$ is given by

$$
\begin{aligned}
\delta\left(\sigma_{\alpha}(\mathcal{A})\right) & =\frac{1}{2^{n}\left|\mathcal{N}_{\mathbb{L}}(\alpha) \mathcal{D}_{\mathbb{L}}\right|^{\frac{1}{2}}} \frac{t_{\alpha}^{n / 2}}{\mathcal{N}_{\mathbb{L}}(\mathcal{A})} \\
& =\frac{1}{2 \sqrt{3}},
\end{aligned}
$$

which is the optimal center density for this dimension, i.e., with the same center density of the lattice $\Lambda_{2}$.

Similarly, in the next table, we have that the lattice $\sigma_{\alpha}(\mathcal{A})$, where $\mathcal{A}$ is an ideal of $\mathcal{O}_{\mathbb{L}}=\mathbb{Z}\left[\zeta_{6}\right]$, has the same center density that the hexagonal lattice $A_{2} \simeq \Lambda_{2}$. 


\begin{tabular}{|c|c|c|c|c|}
\hline \hline $\mathcal{A}$ & $\mathcal{N}_{\mathbb{L}}(\mathcal{A})$ & $\alpha$ & $\mathcal{N}_{\mathbb{L}}(\alpha)$ & $t_{\alpha}$ \\
\hline \hline$\pm \mathcal{O}_{\mathbb{L}}, \pm \zeta_{6} \mathcal{O}_{\mathbb{L}}, \pm\left(1-\zeta_{6}\right) \mathcal{O}_{\mathbb{L}}$ & 1 & 4 & 2 & 4 \\
\hline$\pm\left(2-\zeta_{6}\right) \mathcal{O}_{\mathbb{L}}, \pm\left(1-2 \zeta_{6}\right) \mathcal{O}_{\mathbb{L}}$ & 3 & 4 & 16 & 24 \\
\hline $\pm 2 \mathcal{O}_{\mathbb{L}}, \pm 2 \zeta_{6} \mathcal{O}_{\mathbb{L}}, \pm\left(2-2 \zeta_{6}\right) \mathcal{O}_{\mathbb{L}}$ & 4 & 5 & 25 & 40 \\
\hline$\pm\left(3-2 \zeta_{6}\right) \mathcal{O}_{\mathbb{L}}, \pm\left(1+2 \zeta_{6}\right) \mathcal{O}_{\mathbb{L}}$ & 7 & 3 & 9 & 42 \\
\hline \hline
\end{tabular}

Example 3.5 [12]. The polynomial $p(x)=x^{3}-6 x^{2}+9 x-1$ is irreducible over $\mathbb{Q}$. Since $p(0)=-1, p(1)=5, p(3)=-1$ and $p(4)=3$, it follows that the roots of $p(x)$ are reals. Let $x_{1}, x_{2}$ and $x_{3}$ be the roots of $p(x)$ and $\mathbb{L}=\mathbb{Q}\left(x_{1}\right)$. If $\Lambda$ is a lattice with basis $e_{1}=\left(x_{1}, x_{2}, x_{3}\right), e_{2}=\left(x_{3}, x_{1}, x_{2}\right)$ and $e_{3}=\left(x_{2}, x_{3}, x_{1}\right)$, then

$$
M=\left(\begin{array}{lll}
x_{1} & x_{2} & x_{3} \\
x_{3} & x_{1} & x_{2} \\
x_{2} & x_{3} & x_{1}
\end{array}\right)
$$

is the generator matrix of the lattice $\Lambda$ and $\operatorname{det}(M)=54$. Furthermore, if $x \in \Lambda$ then $x=a_{1} e_{1}+a_{2} e_{2}+a_{3} e_{3}$, with $a_{1}, a_{2}, a_{3} \in \mathbb{Z}$. Thus $|x|^{2}=18\left(a_{1}^{2}+a_{2}^{2}+\right.$ $\left.a_{3}^{2}+a_{1} a_{2}+a_{1} a_{3}+a_{2} a_{3}\right)$, and therefore $t=\min \{|x| ; x \in \Lambda, x \neq 0\}=\sqrt{18}$, with $a_{1}=1$ and $a_{2}=a_{3}=0$. Hence the center density of the lattice $\Lambda$ is given by

$$
\delta(\Lambda)=\left(\frac{t}{2}\right)^{3} \frac{1}{|\operatorname{det}(M)|}=\frac{1}{4 \sqrt{2}},
$$

which is the optimal center density for this dimension, i.e., with the same center density of the lattice $\Lambda_{3}$. In general, if $p(x)=x^{3}+a x^{2}+b x+c$ is irreducible over $\mathbb{Q}$, where $a, b, c \in \mathbb{Z}, a^{2}=4 b$ and $c\left(27 c+4 a^{3}-18 a b\right)<0$, then the lattice $\Lambda$ has the same center density that the lattice $A_{3} \simeq D_{3} \simeq \Lambda_{3}$.

Example 3.6. If $\mathbb{L}=\mathbb{Q}\left(\zeta_{8}\right)$, where $\zeta_{8}$ is a primitive 8-th root of unity, $\mathcal{A}=$ $\left(\zeta_{8}+\zeta_{8}^{2}\right) \mathcal{O}_{\mathbb{L}}$ is an ideal of $\mathcal{O}_{\mathbb{L}}=\mathbb{Z}\left[\zeta_{8}\right]$ and $\alpha=3-2\left(\zeta_{8}+\zeta_{8}^{-1}\right) \in \mathcal{O}_{\mathbb{L}}$, then $[\mathbb{L}: \mathbb{Q}]=4, \mathcal{D}_{\mathbb{L}}=256, \mathcal{N}_{\mathbb{L}}(\mathcal{A})=2$ and $\mathcal{N}_{\mathbb{L}}(\alpha)=1$. If $x \in \mathcal{A}$ then $x=\left(\zeta_{8}+\zeta_{8}^{2}\right)\left(a_{0}+a_{1} \zeta_{8}+a_{2} \zeta_{8}^{2}+a_{3} \zeta_{8}^{3}\right)$, with $a_{0}, a_{1}, a_{2}, a_{3} \in \mathbb{Z}$, and thus

$$
\operatorname{Tr}_{\mathbb{L}}(\alpha x \bar{x})=8\left(a_{0}^{2}-a_{0} a_{1}+a_{1}^{2}-a_{1} a_{2}+a_{2}^{2}+a_{0} a_{3}-a_{2} a_{3}+a_{3}^{2}\right) .
$$


Hence $t_{\alpha}=\min \left\{\operatorname{Tr}_{\mathbb{L}}(\alpha x \bar{x}): x \in \mathcal{A}, x \neq 0\right\}=8$, with $a_{0}=1$ and $a_{1}=a_{2}=$ $a_{3}=0$, and therefore the center density of the lattice $\sigma_{\alpha}(\mathcal{A})$ is given by

$$
\delta\left(\sigma_{\alpha}(\mathcal{A})\right)=\frac{1}{2^{n}\left|\mathcal{N}_{\mathbb{L}}(\alpha) \mathcal{D}_{\mathbb{L}}\right|^{\frac{1}{2}}} \frac{t_{\alpha}{ }^{n / 2}}{\mathcal{N}_{\mathbb{L}}(\mathcal{A})}=\frac{1}{8},
$$

which is the optimal center density for this dimension, i.e., with the same center density of lattice $\Lambda_{4}$.

Similarly, in the next table, we have that the lattice $\sigma_{\alpha}(\mathcal{A})$, where $\mathcal{A}$ is an ideal of $\mathcal{O}_{\mathbb{L}}=\mathbb{Z}\left[\zeta_{8}\right]$, has the same center density that the lattice $D_{4} \simeq \Lambda_{4}$.

\begin{tabular}{|c|c|c|c|c|}
\hline \hline $\mathcal{A}$ & $\mathcal{N}_{\mathbb{L}}(\mathcal{A})$ & $\alpha$ & $\mathcal{N}_{\mathbb{L}}(\alpha)$ & $t_{\alpha}$ \\
\hline \hline $\begin{array}{c} \pm\left(-1+\zeta_{8}{ }^{2}+\zeta_{8}{ }^{3}\right) \mathcal{O}_{\mathbb{L}}, \\
\pm\left(1+\zeta_{8}-\zeta_{8}{ }^{3}\right) \mathcal{O}_{\mathbb{L}}\end{array}$ & 1 & $\begin{array}{c}2-\left(\zeta_{8}+\zeta_{8}^{-1}\right), \\
10-7\left(\zeta_{8}+\zeta_{8}{ }^{-1}\right)\end{array}$ & 4 & 8 \\
\hline $\begin{array}{c} \pm\left(1-2 \zeta_{8}+2 \zeta_{8}{ }^{2}-\zeta_{8}{ }^{3}\right) \mathcal{O}_{\mathbb{L}}, \\
\pm\left(\zeta_{8}-\zeta_{8}{ }^{2}\right) \mathcal{O}_{\mathbb{L}}\end{array}$ & 2 & $6+4\left(\zeta_{8}+\zeta_{8}^{-1}\right)$ & 16 & 16 \\
\hline $\begin{array}{c} \pm\left(1+\zeta_{8}{ }^{2}\right) \mathcal{O}_{\mathbb{L}}, \\
\pm\left(\zeta_{8}-2 \zeta_{8}{ }^{2}+\zeta_{8}{ }^{3}\right) \mathcal{O}_{\mathbb{L}}\end{array}$ & 4 & $2+\left(\zeta_{8}+\zeta_{8}^{-1}\right)$, & 4 & 16 \\
\hline $\begin{array}{c} \pm\left(1+\zeta_{8}+\zeta_{8}{ }^{2}-3 \zeta_{8}{ }^{3}\right) \mathcal{O}_{\mathbb{L}}, \\
\pm\left(1-\zeta_{8}+\zeta_{8}{ }^{2}-\zeta_{8}{ }^{3}\right) \mathcal{O}_{\mathbb{L}}\end{array}$ & 8 & $6+4\left(\zeta_{8}+\zeta_{8}{ }^{-1}\right)$ & 16 & 32 \\
\hline $\begin{array}{c} \pm\left(\zeta_{8}-\zeta_{8}{ }^{2}-\zeta_{8}{ }^{3}\right) \mathcal{O}_{\mathbb{L}}, \\
\pm\left(2-2 \zeta_{8}+\zeta_{8}{ }^{3}\right) \mathcal{O}_{\mathbb{L}}\end{array}$ & 9 & $\begin{array}{c}2+\left(\zeta_{8}+\zeta_{8}^{-1}\right), \\
10+7\left(\zeta_{8}+\zeta_{8}^{-1}\right)\end{array}$ & 4 & 24 \\
\hline $\begin{array}{c} \pm\left(2+2 \zeta_{8}-\zeta_{8}{ }^{3}\right) \mathcal{O}_{\mathbb{L}}, \\
\pm\left(2 \zeta_{8}+2 \zeta_{8}{ }^{2}+2 \zeta_{8}{ }^{3}\right) \mathcal{O}_{\mathbb{L}}\end{array}$ & 16 & $\begin{array}{c}2-\left(\zeta_{8}+\zeta_{8}^{-1}\right), \\
10-7\left(\zeta_{8}+\zeta_{8}^{-1}\right)\end{array}$ & 4 & 32 \\
\hline \hline
\end{tabular}

Example 3.7. If $\mathbb{L}=\mathbb{Q}\left(\zeta_{9}\right)$, where $\zeta_{9}$ is a primitive 9-th root of unity, $\mathcal{A}=$ $\left(1-\zeta_{9}-\zeta_{9}{ }^{2}-\zeta_{9}{ }^{4}-\zeta_{9}{ }^{5}\right) \mathcal{O}_{\mathbb{L}}$ is an ideal of $\mathcal{O}_{\mathbb{L}}=\mathbb{Z}\left[\zeta_{9}\right]$ and $\alpha=4+2 \zeta_{9}{ }^{2}+2 \zeta_{9}{ }^{-2} \in$ $\mathcal{O}_{\mathbb{L}}$, then $n=[\mathbb{L}: \mathbb{Q}]=6, \mathcal{D}_{\mathbb{L}}=3^{9}, \mathcal{N}_{\mathbb{L}}(\mathcal{A})=9$ and $\mathcal{N}_{\mathbb{L}}(\alpha)=64$. If $x \in \mathcal{A}$ then $x=\left(1-\zeta_{9}-\zeta_{9}{ }^{2}-\zeta_{9}{ }^{4}-\zeta_{9}{ }^{5}\right)\left(a_{0}+a_{1} \zeta_{9}+a_{2} \zeta_{9}{ }^{2}+a_{3} \zeta_{9}{ }^{3}+a_{4} \zeta_{9}{ }^{4}+a_{5} \zeta_{9}{ }^{5}\right)$, where $a_{0}, \cdots, a_{5} \in \mathbb{Z}$, and thus $\operatorname{Tr}_{\mathbb{L}}(\alpha x \bar{x})=72 a_{0}^{2}+72 a_{0} a_{1}+72 a_{1}^{2}+36 a_{0} a_{2}+$ $72 a_{1} a_{2}+72 a_{2}^{2}-72 a_{0} a_{3}+36 a_{1} a_{3}+72 a_{2} a_{3}+72 a_{3}^{2}-108 a_{0} a_{4}-72 a_{1} a_{4}+36 a_{2} a_{4}+$ $72 a_{3} a_{4}+72 a_{4}^{2}-108 a_{0} a_{5}-108 a_{1} a_{5}-72 a_{2} a_{5}+36 a_{3} a_{5}+72 a_{4} a_{5}+72 a_{5}^{2}$. 
Hence $t_{\alpha}=\min \left\{\operatorname{Tr}_{\mathbb{L}}(\alpha x \bar{x}): x \in \mathcal{A}, x \neq 0\right\}=36$, with $a_{0}=a_{4}=-1$, $a_{1}=a_{3}=a_{5}=0$ and $a_{2}=1$, and therefore the center density of the lattice $\sigma_{\alpha}(\mathcal{A})$ is given by

$$
\delta\left(\sigma_{\alpha}(\mathcal{A})\right)=\frac{1}{2^{n}\left|\mathcal{N}_{\mathbb{L}}(\alpha) \mathcal{D}_{\mathbb{L}}\right|^{\frac{1}{2}}} \frac{t_{\alpha}{ }^{n / 2}}{\mathcal{N}_{\mathbb{L}}(\mathcal{A})}=\frac{1}{8 \sqrt{3}},
$$

which is the optimal center density for this dimension, i.e., with the same center density of the lattice $\Lambda_{6}$.

Similarly, in the next table, we have that the lattice $\sigma_{\alpha}(\mathcal{A})$, where $\mathcal{A}$ is an ideal of $\mathcal{O}_{\mathbb{L}}=\mathbb{Z}\left[\zeta_{9}\right]$, has the same center density that the lattice $E_{6} \simeq \Lambda_{6}$.

\begin{tabular}{|c|c|c|c|c|}
\hline \hline $\mathcal{A}$ & $\mathcal{N}_{\mathbb{L}}(\mathcal{A})$ & $\alpha$ & $\mathcal{N}_{\mathbb{L}}(\alpha)$ & $t_{\alpha}$ \\
\hline \hline$\pm\left(1-\zeta_{9}{ }^{4}-\zeta_{9}{ }^{5}\right) \mathcal{O}_{\mathbb{L}}$ & 1 & $3-\left(\zeta_{9}+\zeta_{9}{ }^{-1}\right)+\left(\zeta_{9}{ }^{2}+\zeta_{9}{ }^{-2}\right)$ & 81 & 18 \\
\hline$\pm\left(\zeta_{9}{ }^{2}+\zeta_{9}{ }^{4}+\zeta_{9}{ }^{5}\right) \mathcal{O}_{\mathbb{L}}$ & 3 & $5-3\left(\zeta_{9}+\zeta_{9}{ }^{-1}\right)+2\left(\zeta_{9}{ }^{2}+\zeta_{9}{ }^{-2}\right)$ & 9 & 18 \\
\hline$\pm\left(\zeta_{9}{ }^{3}+\zeta_{9}{ }^{4}+\zeta_{9}{ }^{5}\right) \mathcal{O}_{\mathbb{L}}$ & 9 & $2-\left(\zeta_{9}+\zeta_{9}{ }^{-1}\right)-\left(\zeta_{9}{ }^{2}+\zeta_{9}{ }^{-2}\right)$ & 1 & 18 \\
\hline$\pm\left(1+\zeta_{9}-\zeta_{9}{ }^{3}-\zeta_{9}{ }^{4}\right) \mathcal{O}_{\mathbb{L}}$ & 27 & $3-\left(\zeta_{9}+\zeta_{9}{ }^{-1}\right)-2\left(\zeta_{9}{ }^{2}+\zeta_{9}{ }^{-2}\right)$ & 81 & 54 \\
\hline$\pm\left(1-\zeta_{9}-\zeta_{9}{ }^{3}+\zeta_{9}{ }^{4}\right) \mathcal{O}_{\mathbb{L}}$ & 81 & $5-3\left(\zeta_{9}+\zeta_{9}{ }^{-1}\right)+2\left(\zeta_{9}{ }^{2}+\zeta_{9}{ }^{-2}\right)$ & 9 & 54 \\
\hline \hline
\end{tabular}

Example 3.8. If $\mathbb{L}=\mathbb{Q}\left(\zeta_{20}\right)$, where $\zeta_{20}$ is a primitive 20-th root of unity, $\mathcal{A}=\left(2+2 \zeta_{20}-\zeta_{20}^{3}+\zeta_{20}^{4}+\zeta_{20}^{5}-\zeta_{20}^{6}-\zeta_{20}^{7}\right) \mathcal{O}_{\mathbb{L}}$ is an ideal of $\mathcal{O}_{\mathbb{L}}=\mathbb{Z}\left[\zeta_{20}\right]$ and $\alpha=5+5\left(\zeta_{20}+\zeta_{20}^{-1}\right)+5\left(\zeta_{20}^{2}+\zeta_{20}^{-2}\right)+3\left(\zeta_{20}^{3}+\zeta_{20}^{-3}\right) \in \mathcal{O}_{\mathbb{L}}$, then $n=[\mathbb{L}: \mathbb{Q}]=8$, $\mathcal{D}_{\mathbb{L}}=2^{8} 5^{6}, \mathcal{N}_{\mathbb{L}}(\mathcal{A})=16$ and $\mathcal{N}_{\mathbb{L}}(\alpha)=25$. If $x \in \mathcal{A}$ then $x=\left(2+2 \zeta_{20}-\right.$ $\left.\zeta_{20}^{3}+\zeta_{20}^{4}+\zeta_{20}^{5}-\zeta_{20}^{6}-\zeta_{20}^{7}\right)\left(a_{0}+a_{1} \zeta_{20}+a_{2} \zeta_{20}^{2}+a_{3} \zeta_{20}^{3}+a_{4} \zeta_{20}^{4}+a_{5} \zeta_{20}^{5}+a_{6} \zeta_{20}^{6}+a_{7} \zeta_{20}^{7}\right)$, where $a_{0}, \cdots, a_{7} \in \mathbb{Z}$, and thus $\operatorname{Tr}_{\mathbb{L}}(\alpha x \bar{x})=1552 a_{0}^{2}+2944 a_{0} a_{1}+1552 a_{1}^{2}+$ $2496 a_{0} a_{2}+2944 a_{1} a_{2}+1552 a_{2}^{2}+1808 a_{0} a_{3}+2496 a_{1} a_{3}+2944 a_{2} a_{3}+1552 a_{3}^{2}+$ $944 a_{0} a_{4}+1808 a_{1} a_{4}+2496 a_{2} a_{4}+2944 a_{3} a_{4}+1552 a_{4}^{2}+944 a_{1} a_{5}+1808 a_{2} a_{5}+$ $2496 a_{3} a_{5}+2944 a_{4} a_{5}+1552 a_{5}^{2}-944 a_{0} a_{6}+944 a_{2} a_{6}+1808 a_{3} a_{6}+2496 a_{4} a_{6}+$ $2944 a_{5} a_{6}+1552 a_{6}^{2}-1648 a_{0} a_{7}-840 a_{1} a_{7}+32 a_{2} a_{7}+912 a_{3} a_{7}+1704 a_{4} a_{7}+$ $2336 a_{5} a_{7}+2760 a_{6} a_{7}+1360 a_{7}^{2}$. Hence $t_{\alpha}=\min \left\{\operatorname{Tr}_{\mathbb{L}}(\alpha x \bar{x}): x \in \mathcal{A}, x \neq\right.$ $0\}=40$, with $a_{0}=a_{3}=0, a_{1}=a_{5}=a_{6}=-1$ and $a_{2}=a_{4}=a_{7}=1$, and therefore the center density of the lattice $\sigma_{\alpha}(\mathcal{A})$ is given by

$$
\delta\left(\sigma_{\alpha}(\mathcal{A})\right)=\frac{1}{2^{n}\left|\mathcal{N}_{\mathbb{L}}(\alpha) \mathcal{D}_{\mathbb{L}}\right|^{\frac{1}{2}}} \frac{t_{\alpha}{ }^{n / 2}}{\mathcal{N}_{\mathbb{L}}(\mathcal{A})}=\frac{1}{16},
$$


which is the optimal center density for this dimension, i.e., with the same center density of the lattice $\Lambda_{8}$.

Similarly, in the next table, we have that the lattice $\sigma_{\alpha}(\mathcal{A})$, where $\mathcal{A}$ is an ideal of $\mathcal{O}_{\mathbb{L}}=\mathbb{Z}\left[\zeta_{20}\right]$, has the same center density that the lattice $E_{8} \simeq \Lambda_{8}$.

\begin{tabular}{|c|c|c|c|c|}
\hline \hline $\mathcal{A}$ & $\mathcal{N}_{\mathbb{L}}(\mathcal{A})$ & $\alpha$ & $\mathcal{N}_{\mathbb{L}}(\alpha)$ & $t_{\alpha}$ \\
\hline \hline $\begin{array}{c} \pm\left(1-2 \zeta_{20}-\zeta_{20}^{5}+2 \zeta_{20}^{7}\right) \mathcal{O}_{\mathbb{L}}, \\
\pm\left(1-\zeta_{20}^{3}+\zeta_{20}^{4}-\zeta_{20}^{6}-\zeta_{20}^{7}\right) \mathcal{O}_{\mathbb{L}}\end{array}$ & 16 & $\begin{array}{c}2-\left(\zeta_{20}+\zeta_{20}^{-1}\right) \\
+\left(\zeta_{20}^{2}+\zeta_{20}^{-2}\right)\end{array}$ & 25 & 40 \\
\hline $\begin{array}{c} \pm\left(2-\zeta_{20}^{2}+2 \zeta_{20}^{4}-2 \zeta_{20}^{6}+\zeta_{20}^{7}\right) \mathcal{O}_{\mathbb{L}}, \\
\pm\left(1-\zeta_{20}^{2}-\zeta_{20}^{3}-\zeta_{20}^{4}-\zeta_{20}^{6}\right) \mathcal{O}_{\mathbb{L}}\end{array}$ & 80 & $3-\left(\zeta_{20}^{2}+\zeta_{20}^{-2}\right)$ & 1 & 40 \\
\hline $\begin{array}{c} \pm\left(2+\zeta_{20}+2 \zeta_{20}^{4}-\zeta_{20}^{6}\right) \mathcal{O}_{\mathbb{L}}, \\
\pm\left(1+\zeta_{20}+\zeta_{20}^{2}+\zeta_{20}^{3}+\zeta_{20}^{4}\right) \mathcal{O}_{\mathbb{L}}\end{array}$ & 256 & $\begin{array}{c}2-\left(\zeta_{20}+\zeta_{20}^{-1}\right) \\
+\left(\zeta_{20}^{2}+\zeta_{20}^{-2}\right)\end{array}$ & 25 & 40 \\
\hline \hline
\end{tabular}

Example 3.9. If $\mathbb{L}=\mathbb{Q}\left(\zeta_{21}\right)$, where $\zeta_{21}$ is a primitive 21-th root of unity, $\mathcal{A}=$ $\left(\zeta_{21}^{2}-\zeta_{21}^{4}+\zeta_{21}^{8}\right) \mathcal{O}_{\mathbb{L}}$ is an ideal of $\mathcal{O}_{\mathbb{L}}=\mathbb{Z}\left[\zeta_{21}\right]$ and $\alpha=1$, then $n=[\mathbb{L}: \mathbb{Q}]=12$, $\mathcal{D}_{\mathbb{L}}=3^{6} 7^{10}, \mathcal{N}_{\mathbb{L}}(\mathcal{A})=7$. If $x \in \mathcal{A}$ then $x=\left(\zeta_{21}^{2}-\zeta_{21}^{4}+\zeta_{21}^{8}\right)\left(a_{0}+a_{1} \zeta_{21}+\right.$ $\left.a_{2} \zeta_{21}^{2}+a_{3} \zeta_{21}^{3}+a_{4} \zeta_{21}^{4}+a_{5} \zeta_{21}^{5}+a_{6} \zeta_{21}^{6}+a_{7} \zeta_{21}^{7}+a_{8} \zeta_{21}^{8}+a_{9} \zeta_{21}^{9}+a_{10} \zeta_{21}^{10}+a_{11} \zeta_{21}^{11}\right)$ where $a_{0}, \cdots, a_{11} \in \mathbb{Z}$, and thus $\operatorname{Tr}_{\mathbb{L}}(\alpha x \bar{x})=28 a_{0}^{2}+28 a_{1}^{2}+28 a_{0} a_{10}-14 a_{1} a_{10}+$ $28 a_{10}^{2}+28 a_{0} a_{11}+28 a_{1} a_{11}+28 a_{11}^{2}-14 a_{0} a_{2}-14 a_{11} a_{2}+28 a_{2}^{2}-14 a_{0} a_{3}-14 a_{1} a_{3}-$ $28 a_{10} a_{3}+28 a_{3}^{2}-14 a_{0} a_{4}-14 a_{1} a_{4}-28 a_{11} a_{4}-14 a_{2} a_{4}+28 a_{4}^{2}+28 a_{0} a_{5}-14 a_{1} a_{5}+$ $28 a_{10} a_{5}-14 a_{2} a_{5}-14 a_{3} a_{5}+28 a_{5}^{2}+28 a_{1} a_{6}-14 a_{10} a_{6}+28 a_{11} a_{6}-14 a_{2} a_{6}-$ $14 a_{3} a_{6}-14 a_{4} a_{6}+28 a_{6}^{2}-28 a_{0} a_{7}-14 a_{10} a_{7}-14 a_{11} a_{7}+28 a_{2} a_{7}-14 a_{3} a_{7}-$ $14 a_{4} a_{7}-14 a_{5} a_{7}+28 a_{7}^{2}-28 a_{1} a_{8}-14 a_{10} a_{8}-14 a_{11} a_{8}+28 a_{3} a_{8}-14 a_{4} a_{8}-$ $14 a_{5} a_{8}-14 a_{6} a_{8}+28 a_{8}^{2}-14 a_{0} a_{9}-14 a_{11} a_{9}-28 a_{2} a_{9}+28 a_{4} a_{9}-14 a_{5} a_{9}-$ $14 a_{6} a_{9}-14 a_{7} a_{9}+28 a_{9}^{2}$. Hence $t_{\alpha}=\min \left\{\operatorname{Tr}_{\mathbb{L}}(\alpha x \bar{x}): x \in \mathcal{A}, x \neq 0\right\}=28$, with $a_{0}=a_{1}=a_{2}=a_{3}=a_{4}=a_{5}=a_{6}=a_{8}=a_{9}=a_{10}=a_{11}=0$ and $a_{7}=-1$, and therefore the center density of the lattice $\sigma_{\alpha}(\mathcal{A})$ is given by

$$
\delta\left(\sigma_{\alpha}(\mathcal{A})\right)=\frac{1}{2^{n}\left|\mathcal{N}_{\mathbb{L}}(\alpha) \mathcal{D}_{\mathbb{L}}\right|^{\frac{1}{2}}} \frac{t_{\alpha}{ }^{n / 2}}{\mathcal{N}_{\mathbb{L}}(\mathcal{A})}=\frac{1}{27},
$$

which is the optimal center density for this dimension, i.e., with the same center density of the lattice $K_{12}$. 
Similarly, in the next table, we have that the lattice $\sigma_{\alpha}(\mathcal{A})$, where $\mathcal{A}$ is an ideal of $\mathcal{O}_{\mathbb{L}}=\mathbb{Z}\left[\zeta_{21}\right]$ and $\alpha=1$, has the same center density that the lattice $K_{12}$.

\begin{tabular}{|c|c|c|}
\hline \hline $\mathcal{A}$ & $\mathcal{N}(\mathcal{A})$ & $t_{\alpha}$ \\
\hline \hline$\pm\left(1+\zeta_{21}^{6}-\zeta_{21}^{8}\right) \mathcal{O}_{\mathbb{L}}, \pm\left(1-\zeta_{21}^{4}+\zeta_{21}^{6}\right) \mathcal{O}_{\mathbb{L}}$, & & \\
$\pm\left(1-\zeta_{21}^{2}+\zeta_{21}^{6}\right) \mathcal{O}_{\mathbb{L}}, \pm\left(1-\zeta_{21}^{2}-\zeta_{21}^{8}\right) \mathcal{O}_{\mathbb{L}}$, & 7 & 28 \\
$\left(-\zeta_{21}^{2}+\zeta_{21}^{4}-\zeta_{21}^{8}\right) \mathcal{O}_{\mathbb{L}}$ & & \\
\hline \hline
\end{tabular}

\section{Conclusions}

In this work we presented examples of algebraic lattices via the twisted canonical homomorphism with optimal center density in dimensions 2, 3, 4, 6, 8 and 12. These algebraic lattices are rotated versions of known dense lattices. Note that the examples given in this work are not new either. What is new however is the way the densities of the lattices are checked through computations rather than by theoretic arguments. Furthermore, with the use of canonical homomorphism we believe that it is possible to construct algebraic lattices with optimal center density in other dimensions.

Acknowledgements. The authors would like to thank the anonymous reviewers for their insightful comments that greatly improved the quality of this work.

\section{REFERENCES}

[1] E. Bayer-Fluckiger, Definite unimodular lattices having an automorphism of given characteristic polynomial. Comment. Math. Helvetici, 59 (1984), 509-538.

[2] E. Bayer-Fluckiger and J. Martinet, Formes quadratiques liées aux algèbres semi-simples. J. Reine Angew. Math., 451 (1994), 51-69.

[3] E. Bayer-Fluckiger, Lattices and number fields. Contemporany Mathematics, 241 (1999), 69-84.

[4] E. Bayer-Fluckiger, Cyclotomic modular lattices. Journal de Théorie des Nombres de Bordeaux, 12 (2000), 273-280.

[5] E. Bayer-Fluckiger, Ideal lattices. Proceedings of the conference number theory and diophantine geometry, (Zurich, 1999), Cambridge Univ. Press (2002), 168-184.

[6] E. Bayer-Fluckiger, Modular lattices over cyclotomic fields. Journal of Number Theory, 114 (2005), 394-411. 
[7] E. Bayer-Fluckiger, Ideal lattices over totally real number fields and euclidian minima. Archiv Math., 86 (2006), 217-225.

[8] X. Giraud and J.C. Belfiore, Constellations matched to the Rayleigh fading channel. IEEE Trans. on Inform. Theory, 42-1 (1996), 106-115.

[9] J. Boutros, E. Viterbo, C. Rastello and J-C. Belfiore, Good lattice constellations for both Rayleigh fading and Gaussian channels. IEEE Trans. Inform. Theory, 42-2 (1996), 502-517.

[10] P. Samuel, Algebraic theory of numbers. Hermann, Paris (1970).

[11] J.H. Conway and N.J.A. Sloane, Sphere packing, lattices and groups. Springer-Verlag, New York (1999).

[12] T.M. Souza, Algebraic lattices in Abelian number field. M.D. Dissertation, Ibilce-Unesp, São José do Rio Preto, SP (2004). 